\title{
Platelet-derived growth factor receptor alpha-positive cells: a new cell type in the human ureteropelvic junction
}

\author{
Manuela Hunziker ${ }^{1}$, Anne-Marie O'Donnell ${ }^{1}$ and Prem Puri ${ }^{1}$
}

BACKGROUND:: Ureteropelvic junction (UPJ) obstruction is the most common cause of congenital hydronephrosis. Normal ureteral motility requires coordinated interaction between neurons, smooth muscle cells (SMCs), and interstitial Cajal-like cells (IC-LCS). Recently, a new type of interstitial cell, platelet-derived growth factor receptor a-positive (PDGFRa $\left.{ }^{+}\right)$ cells, was discovered in the gastrointestinal tract and bladder. METHODS: We used immunohistochemistry to study PDGFRa protein distribution in normal human UPJ and congenital UPJ obstruction. Western blot and real-time PCR (RT-PCR) were used to study PDGFRa protein and gene expression levels. In addition, closely associated cells and small conductance $\mathrm{Ca}^{2}$ ${ }^{+}$-activated $\mathrm{K}^{+}(\mathrm{SK})$ channels were investigated.

RESULTS: PDGFRa ${ }^{+}$cells were distinct from IC-LCs and SMCs and were in close proximity to nerve fibers. PDGFRa ${ }^{+}$cells expressed SK3 channels, which are thought to mediate purinergic inhibitory neurotransmission in SMCs. The distribution of PDGFRa ${ }^{+}$cells was similar in UPJ obstruction vs. controls. However, the expression of SK3 channels in PDGFRa ${ }^{+}$ cells was decreased in UPJ obstruction vs. controls.

CONCLUSION: This study shows, for the first time, the $\mathrm{PDGFRa}^{+}$cell expression in the human UPJ. Altered SK3 channel expression observed in PDGFRa ${ }^{+}$cells in UPJ obstruction suggests that the impairment of SK3 activity across the UPJ may perturb upper urinary tract peristalsis in this urological condition.

$\mathbf{U}$ reteropelvic junction (UPJ) obstruction is the most common cause of congenital hydronephrosis in children occurring in 1 in 1,000-2,000 live births (1). Although the exact mechanisms of pelviureteral peristalsis are poorly understood, it is known that pacemaker cells regulate the coordinated contraction of the renal pelvis and ureter to propel urine from the kidney to the bladder. The following three types of electrically active cells have been identified in the UPJ: atypical smooth muscle cells (SMCs), typical SMCs, and interstitial Cajal-like cells (IC-LCs) (2). Failure of transmission of peristaltic waves across the UPJ leads to the accumulation of urine in the kidney and dilatation of the renal pelvis in UPJ obstruction. UPJ obstruction has been classified into extrinsic and intrinsic pathologic abnormalities. Extrinsic obstruction may result from mechanical factors such as aberrant crossing vessels, fibrous band adhesions, or kinks causing angulations or compressions. The nature of the abnormality in the more common intrinsic UPJ obstruction is not fully understood. Previous studies have demonstrated abnormalities in the UPJ including abnormal innervation patterns $(3,4)$, hypotrophy/hypertrophy of the smooth muscle in the obstructive segment with muscular derangement, and its replacement with collagen (1), as well as altered expression of IC-LCs (5).

To date, two types of interstitial cells have been described in the upper urinary tract using antibodies against the tyrosine kinase receptor c-kit: c-kit-positive and c-kit-negative interstitial cells. Different research groups have described them as interstitial cells of Cajal (ICCs), ICC-like cells, interstitial Cajal-like cells (IC-LCs), interstitial cells, myofibroblasts, telocytes, and renal interstitial cell $(2,6,7,8)$. However, there is no general agreement on the nomenclature of these specialized cells. Moreover, although immunolabeling has shown that a complex array of interstitial cells exists within the layers of the UPJ, their structural and molecular expression as well as their functional roles are still largely unknown. Interstitial cells are thought to have a role in the transduction of neural inputs in order to generate electrical activity, which they propagate to SMCs (9).

A new type of interstitial cells, known as "fibroblast-like cells", has been discovered in the gastrointestinal tract, (10) and more recently in the bladder $(11,12)$. These cells have been characterized with an antibody raised against the platelet-derived growth factor receptor alpha (PDGFR $\alpha$ ) protein. These novel interstitial cells, PDGFR $\alpha$-positive $\left(\mathrm{PDGFR}^{+}\right)$cells, have been shown to be at least part of what have been previously known as the c-kit-negative ICLCs (9). In the gastrointestinal tract, PDGFR $\alpha^{+}$cells have been found to be located adjacent to c-kit-positive ICCs and close to enteric nerves and SMCs, suggesting a role for these cells in neurotransmission and neuromodulation (10). Studies in the bladder and gastrointestinal tract have further shown that PDGFR $\alpha^{+}$cells express small conductance $\mathrm{Ca}^{2+}$-activated $\mathrm{K}^{+}$(SK) channels $(13,14)$. Although SK2 channels are the major functional subtype of SK channels in the mouse urinary

\footnotetext{
${ }^{1}$ National Children's Research Centre, Department of Pediatric Surgery, Our Lady's Children's Hospital, Dublin, Ireland. Correspondence: Prem Puri (prem.puri@ucd.ie) Received 17 November 2016; accepted 31 July 2017; advance online publication 13 September 2017. doi:10.1038/pr.2017.193
} 


\section{PDGFR $\alpha^{+}$cells in the human UPJ Articles}

bladder, modulating contractility in SMCs (15), SK3 channels have been shown to mediate purinergic inhibitory neurotransmission (9).

The discovery of PDGFR $\alpha^{+}$cells in the gastrointestinal tract and bladder raises the question of whether these cells are also expressed in the human UPJ. The aim of this study was, therefore, to determine the distribution of PDGFR $\alpha^{+}$cells and their relationship to nerve cells, IC-LCs, and SMCs in the normal human UPJ and congenital UPJ obstruction. Furthermore, we investigated the expression of SK channels in PDGFR $\alpha^{+}$cells and SMCs of the human UPJ.

\section{METHODS}

This study was approved by the Ethics (Medical Research) Committee, Our Lady's Children's Hospital Crumlin, Dublin (Ref. GEN/200/11), and tissue samples were obtained with informed parental consent. Between 2010 and 2014, we prospectively collected UPJ specimens from 28 patients with a mean age of 1.3 years (range 3 months to 3.3 years) who underwent pyeloplasty for intrinsic UPJ obstruction. All cases of intrinsic UPJ obstruction were confirmed by radiological and surgical findings. Extrinsic UPJ obstruction cases were excluded from the study. Control samples included 20 UPJ specimens from patients who underwent Wilm's tumor nephrectomy, with a mean age of 2.4 years (range 4 months to 4.8 years). Patients who underwent Wilm's tumor nephrectomy did not present with any clinical or radiological UPJ obstruction findings, and their UPJs were free of tumor-cell invasion.

Tissue at $1.5 \mathrm{~cm}$ from the UPJ region, containing the UPJ, was resected from control and UPJ obstruction cases. In UPJ controls, we first identified the site where the funnel-shaped renal pelvis became continuous with the ureter and resected $1.5 \mathrm{~cm}$ of the UPJ. In UPJ obstruction cases, the junction was easier to identify because of the abrupt luminal narrowing of the pelvis, and $1.5 \mathrm{~cm}$ of UPJ was obtained. Specimens were either snap-frozen in liquid nitrogen or stored at $-80{ }^{\circ} \mathrm{C}$ for RNA and protein extraction, or were embedded in OCT Mounting Compound (VWR International, Leuven, Belgium) and stored at $-80{ }^{\circ} \mathrm{C}$ for immunofluorescence staining and confocal microscopy.

\section{Immunofluorescence Staining and Confocal Microscopy}

Frozen blocks of three UPJ obstruction and three UPJ control samples were sectioned transversely at a thickness of $10 \mu \mathrm{m}$, mounted on SuperFrost Plus slides (VWR International), and were fixed with $10 \%$ buffered formalin for $5 \mathrm{~min}$. Sections underwent cell membrane permeabilization with $1 \%$ Triton X-100 for 20 min at room temperature. After blocking with $10 \%$ bovine serum albumin (Sigma-Aldrich, Arklow, Ireland) for $30 \mathrm{~min}$ to avoid nonspecific absorption, the sections were incubated overnight at $4{ }^{\circ} \mathrm{C}$ with a mixture of primary antibodies diluted in phosphate-buffered saline with $1 \%$ bovine serum albumin. Information on primary antibody sources and concentrations is shown in Table 1. The sections were then washed in phosphate-buffered saline+0.05\% Tween and incubated with corresponding secondary antibodies or Phalloidin for $1 \mathrm{~h}$ at room temperature. Information on secondary antibody sources and concentrations is shown in Table 1. After washing, the sections were counterstained with a 4',6-diamidino-2-phenylindole antibody (10236276001, dilution 1:1,000, Roche Diagnostics, Mannheim, Germany) for $10 \mathrm{~min}$, washed, mounted, and coverslipped with Fluorescent Mounting Medium (DAKO, Glostrup, Denmark). All sections were independently evaluated by two investigators with a LSM 700 confocal microscope (Carl Zeiss MicroImaging, Jena, Germany).

\section{Western Blot}

A total of nine obstructed UPJ and nine control UPJ specimens were homogenized in RIPA buffer (Radio Immunoprecipitation Assay
Table 1. Antibodies and phalloidin used for immunofluorescence and western blotting

\begin{tabular}{lcccl}
\hline & Species & Catalog no. & Dilution & Source \\
\hline $\begin{array}{l}\text { Primary antibodies } \\
\text { PDGFRa }\end{array}$ & Goat & AF-307-NA & $1: 100$ & RD Systems, USA \\
PDGFRa & Rabbit & ab61219 & $1: 100$ & Abcam, UK \\
c-kit & Rabbit & A4502 & $1: 100$ & Dako, Denmark \\
SK3 (KCNN3) & Goat & Sc-16027 & $1: 100$ & Santa Cruz \\
& & & & Biotechnology, \\
& Mouse & AA301-410 & $1: 100$ & Abnova, Taiwan \\
SK3 (KCNN3) & Goat & ab99457 & $1: 100$ & Abcam, UK \\
SK2 (KCNN2) & Rabbit & Z5116 & $1: 100$ & Dako, Denmark \\
PGP9.5 & & & &
\end{tabular}

\begin{tabular}{|c|c|c|c|}
\hline Secondary antibodies & & $1: 500$ & \\
\hline $\begin{array}{l}\text { Alexa Fluor } 488 \text { donkey } \\
\text { anti-mouse lgG }\end{array}$ & ab150109 & $1: 500$ & Abcam, UK \\
\hline $\begin{array}{l}\text { Alexa Fluor } 555 \text { donkey } \\
\text { anti-goat lgG }\end{array}$ & ab150134 & $1: 500$ & Abcam, UK \\
\hline Alexa Fluor 647 donkey & ab150067 & $1: 500$ & Abcam, UK \\
\hline
\end{tabular}

anti-rabbit IgG

\begin{tabular}{|c|c|c|c|}
\hline $\begin{array}{l}\text { Alexa Fluor } \\
\text { Phalloidin } 635\end{array}$ & A34054 & $1: 500$ & Invitrogen, UK \\
\hline $\begin{array}{l}\text { Alexa Fluor } \\
\text { Phalloidin } 488\end{array}$ & A12379 & $1: 500$ & Invitrogen, UK \\
\hline
\end{tabular}

buffer from Sigma-Aldrich) containing 1\% protease inhibitor cocktail (Sigma-Aldrich). Protein concentrations were determined using a Bradford assay (Sigma-Aldrich). A total volume of $20 \mu \mathrm{l} \mathrm{Laemmli}$ sample buffer (Sigma-Aldrich) containing $10 \mu \mathrm{g}$ of protein was loaded into $10 \%$ sodium dodecyl sulfate-polyacrylamide gel electrophoresis gels (NuPAGE Novex Bis-Tris gels, Invitrogen, Cramlington, UK) for electrophoretic separation. Electrophoresis was performed in MES sodium dodecyl sulfate running buffer (Invitrogen, UK). Proteins were then transferred to $0.45 \mu \mathrm{m}$ nitrocellulose membrane (Merck Millipore, Billerica, MA) by western blotting. Following western blot analysis, the membranes were blocked in 3\% bovine serum albumin$0.05 \%$ Tween for $30 \mathrm{~min}$ before antibody detection. Primary antibodies against c-kit, PDGFR $\alpha$, SK2, SK3, and pan-neuronal marker protein gene product 9.5 (PGP9.5) were used and incubation was performed overnight at $4{ }^{\circ} \mathrm{C}$. Following extensive washing (four times in phosphate-buffered saline $-0.05 \%$ Tween) the membranes were incubated with the appropriate secondary antibodies followed by washing. Information on primary and secondary antibody sources and concentrations is shown in Table 1. Detection was performed with the PIERCE chemiluminescence kit (Thermo Fisher Scientific, Dublin, Ireland). We used $ß$-actin (Anti-beta actin antibody, ab8227, dilution 1:5,000, Abcam, Cambridge, UK) as an additional loading control. Band density was quantified by digital densitometry using the image-analysis software ImageJ (NIH, Bethesda, MD). Band intensity was normalized to that of $\beta$-actin.

\section{RNA Isolation}

Total RNA was isolated using the Qiagen miRNeasy Mini Kit (Qiagen, Manchester, UK) according to the manufacturer's protocol. Spectrophotometrical quantification of total RNA was performed (NanoDrop ND-1000 UV-Vis Spectrophotometer) and the RNA was stored at $-80^{\circ} \mathrm{C}$.

cDNA Synthesis and Quantitative Polymerase Chain Reaction Reverse transcription of total RNA was carried out at $85^{\circ} \mathrm{C}$ for $3 \mathrm{~min}$ (denaturation), at $44{ }^{\circ} \mathrm{C}$ for $60 \mathrm{~min}$ (annealing), and at $92{ }^{\circ} \mathrm{C}$ for 
Table 2. Primers for quantitative RT-PCR

\begin{tabular}{ll}
\hline Gene names & Primer sequence $\left(5^{\prime}-3^{\prime}\right)$ \\
\hline PDGFRafw & CGAGGAAGACAAGCTGAAGG \\
PDGFRarv & AGGGACAGGGTCAATGTCAG \\
PGP9.5fw & AACAATCAGCAATGCCTGTG \\
PGP9.5rv & TCCAGGTATCTGGCTCGTTC \\
SK2fw & GGAGCAGAGGAGACTGAATGAC \\
SK2rv & CAGGGTAACAATCCTCTTCTCG \\
SK3fw & GGGCCCTTGCTGCAGAG \\
SK3rv & CCCGCGTGAAGACCTGAG \\
GAPDHfw & GGAGTCAACGGATTGGT \\
GAPDHrv & GTGATGGGATTCCATTGAT \\
\hline
\end{tabular}

RT-PCR, reverse-transcriptase PCR.

10 min (reverse transcriptase inactivation), using Transcriptor High Fidelity cDNA Synthesis Kit (Roche Diagnostics) according to the manufacturer's instructions. Resulting cDNA was used for quantitative real-time PCR (qRT-PCR) using a LightCycler 480 SYBR Green I Master (Roche Diagnostics) in a total reaction mix of $20 \mu \mathrm{l}$ per well. A total of 16 UPJ samples and 8 control samples were used for RT-PCR. Gene-specific primer pairs are listed in Table 2. After 5 min of initial denaturation at $95{ }^{\circ} \mathrm{C}, 45$ cycles of amplification for each primer were carried out. Each cycle included denaturation at $95^{\circ} \mathrm{C}$ for $10 \mathrm{~s}$, annealing at $60{ }^{\circ} \mathrm{C}$ for $15 \mathrm{~s}$, and elongation at $72{ }^{\circ} \mathrm{C}$ for $10 \mathrm{~s}$. The relative changes in expression levels of PDGFR $\alpha, P G P 9.5$, $S K 2$, and SK3 were normalized against the levels of GAPDH gene expression in each sample $\left(\Delta \Delta \mathrm{C}_{\mathrm{T}}\right.$ method). Experiments were carried out in triplicate for each sample and primer.

\section{Statistical Analysis}

Numerical data are shown as mean \pm SEM. The statistical significance of difference in gene expression levels between UPJ obstruction and controls was determined by Student's $t$-test or Mann-Whitney $U$-test, depending on the distribution of the data. A $P$ value of $<0.05$ was considered statistically significant.

\section{RESULTS}

\section{Confocal Immunofluorescence}

Distribution of PDGFRa ${ }^{+}$cells. The distribution of PDGFR $\alpha^{+}$ cells was examined on cryostat sections of the human UPJ using confocal microscopy. PDGFR $\alpha^{+}$cells were widely distributed and had spindle-shaped or stellate-shaped morphology (Figure 1). These cells formed discrete networks and were observed in the lamina propria and throughout the inner longitudinal and outer circular muscle layers of the human UPJ. The distribution of PDGFR $\alpha^{+}$cells in UPJ obstruction samples and UPJ control samples was similar (data not shown).

Relationship to IC-LCs, SMCs, and nerve cells. To assess whether PDGFR $\alpha^{+}$cells were structurally associated with SMCs, UPJ samples were double-labeled with a PDGFR $\alpha$ antibody and phalloidin, which labels filamentous actin. PDGFR $\alpha^{+}$cells were located close to phalloidin-positive SMCs, but were distinct from SMCs (Figure 2). PDGFR $\alpha^{+}$cells surrounded phalloidinpositive smooth muscle bundles.

C-kit is widely used to label IC-LCs in the upper urinary tract. We, therefore, performed double-labeling immunohistochemistry to examine whether c-kit-positive cells were co-

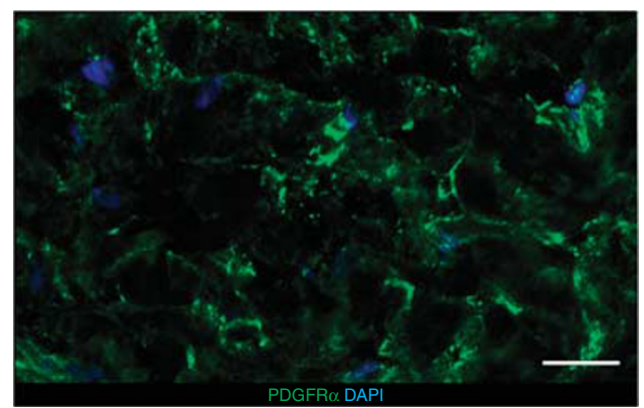

Figure 1. Immunolabeling of PDGFRa (green) in the muscle layers of human control UPJ. PDGFRa+ cells formed an extensive fine network. Nuclei were stained with DAPI (blue). Bar $=20 \mu \mathrm{m}$. DAPI, 4',6-diamidino2-phenylindole; PDGFRa+, platelet-derived growth factor receptor a positive; UPJ, ureteropelvic junction.

localized with PDGFR $\alpha^{+}$cells. PDGFR $\alpha^{+}$cells were found to be adjacent to, but distinct from c-kit-positive IC-LCs (Figure 2). IC-LCs were markedly reduced in UPJ obstruction compared with controls.

In order to determine whether PDGFR $\alpha^{+}$cells were closely associated with nerve cells, we performed double-labeling immunohistochemistry using a PDGFR $\alpha$ antibody and PGP9.5. PDGFR $\alpha^{+}$cells were observed to be in close proximity to PGP9.5-positive nerve cells (Figure 2). In addition, in UPJ obstruction, PGP9.5-positive nerve cells were markedly reduced compared with UPJ controls.

Expression of SK2 and SK3. We then assessed the expression of SK channels in PDGFR $\alpha^{+}$cells and SMCs. Double-labeling immunohistochemistry using SK2, SK3, PDGFR $\alpha$, and Phalloidin antibodies was performed. Confocal-immunofluorescence-double staining revealed strong SK3 expression within a subset of PDGFR $\alpha^{+}$cells (Figure 3). Furthermore, SK3 expression in PDGFR $\alpha^{+}$cells of the human UPJ was decreased in UPJ-obstructed specimens when compared with controls. Confocal-immunofluorescencedouble staining also showed that SK2 was expressed in SMCs, but not in PDGFR $\alpha^{+}$cells. There was an increased SK2 immunoreactivity in SMCs in UPJ obstruction when compared with controls (Figure 4).

\section{Western Blot}

To further consolidate the immunofluorescence results, we investigated protein expression levels using western blot analysis, which revealed expression of PDGFR $\alpha$, PGP9.5, SK2, and SK3 in the human UPJ (Figure 5). PDGFR $\alpha$ protein expression levels in UPJ obstruction were not different from controls. Western blotting confirmed decreased protein expression levels of PGP9.5 and SK3 and increased protein expression levels of SK2 in UPJ obstruction when compared with controls (Figure 5).

\section{Relative mRNA Expression Levels}

To support the western blot results, we performed RT-PCR to detect the relative mRNA expression levels of PDGFR $\alpha$, 

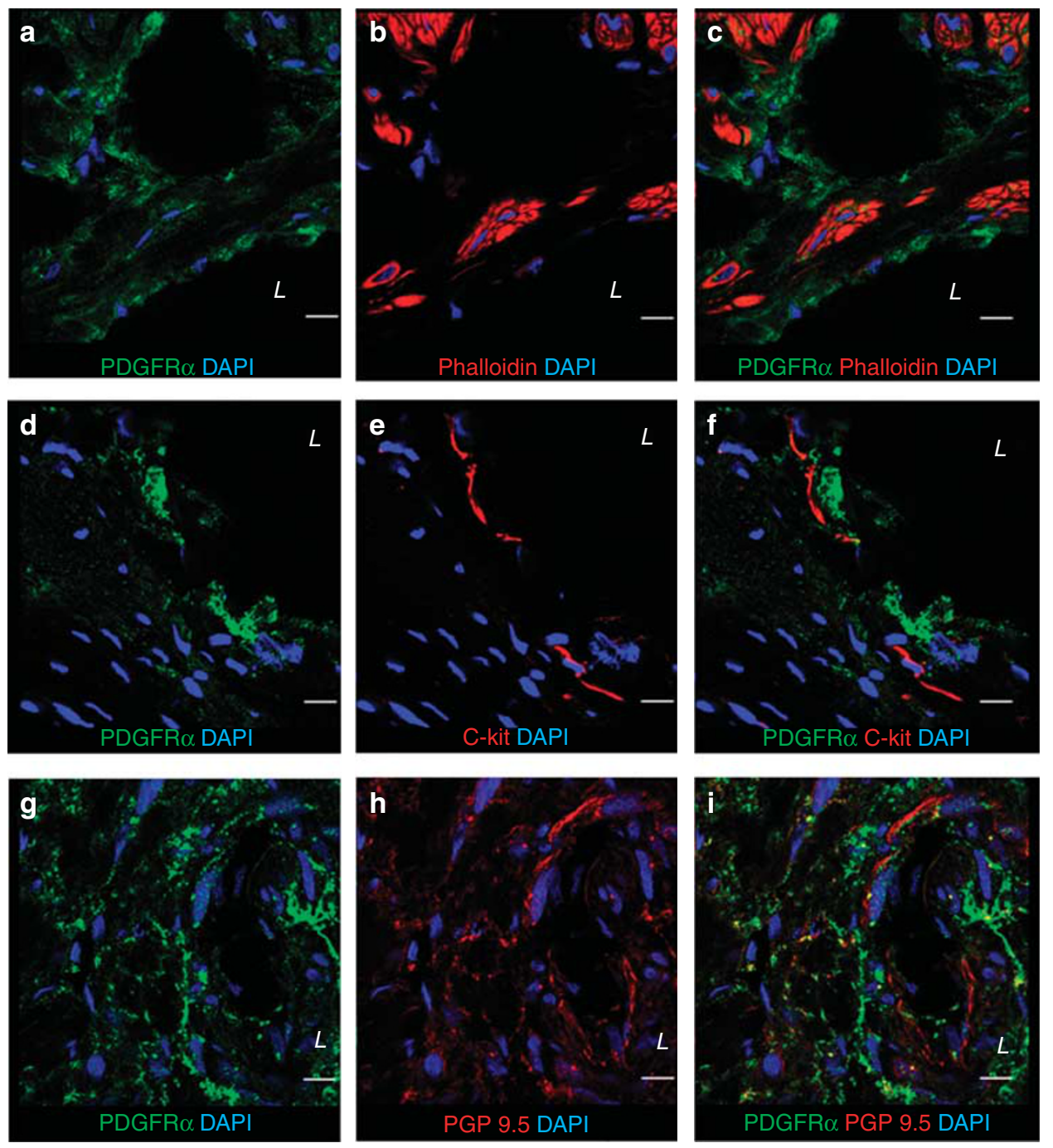

Figure 2. Expression of PDGFRa+ cells, IC-LCS, SMCs and PGP9.5-positive nerve cells. Double immunolabeling of PDGFRa (green) and phalloidin (red) in the muscle layers of the human UPJ control (a-c). PDGFRa+ cells were located close to phalloidin-positive SMCs, but were distinct from SMCs. Double immunolabeling of PDGFRa (green) and c-kit (red) in the muscle layers of the human UPJ control (d-f). PDGFRa+cells were adjacent to, but distinct from c-kit-positive IC-LCs. Double immunolabeling of PDGFRa (green) and PGP9.5 (red) in the muscle layers of the human UPJ control (g-i). PDGFRa+ cells were closely associated with PGP9.5-positive cells and nerve fibers. Nuclei were stained with DAPI (blue) in all panels. $L$ is indicating the luminal side. Bar $=10 \mu \mathrm{m}$ in all panels. DAPI, 4',6-diamidino-2-phenylindole; IC-LC, interstitial Cajal-like cell; PDGFRa+, platelet-derived growth factor receptor a positive; PGP9.5, pan-neuronal marker protein gene product 9.5; SMC, smooth muscle cell; UPJ, ureteropelvic junction.

PGP9.5, SK2, and SK3 channels. RT-PCR revealed the mRNA transcripts for all targets. There was no significant difference between UPJ obstruction and controls in the relative mRNA expression level of PDGFR $\alpha$. The relative mRNA expression levels of PGP9.5 and SK3 were significantly decreased in UPJ obstruction, whereas SK2 gene expression levels were significantly increased compared with controls (Figure 5).

\section{DISCUSSION}

Interstitial cells in the urinary tract have attracted researchers for many years. These cells have important regulatory functions and interact with SMCs. Although interstitial cells in the bladder have been well studied, they are relatively unexplored in the upper urinary tract (16). Labeling with a c-kit antibody is the standard technique for the identification of IC-LCs in the upper urinary tract, and interstitial cells have, therefore, been divided into c-kit-positive and c-kit-negative cells (9). A new type of interstitial cell, PDGFR $\alpha^{+}$cells, was first identified in the murine gastrointestinal tract in 2009 (ref. 10), followed by identification in mouse (12), human, and guinea pig urinary bladders (11).

Our findings indicate the presence of PDGFR $\alpha^{+}$cells in the human UPJ. RT-PCR and western blot confirmed the expression levels of the PDGFR $\alpha$ gene and protein, respectively. Our immunohistochemical studies showed that: (i) PDGFR $\alpha^{+}$cells had spindle-shaped morphology and were distributed throughout the UPJ wall, including the lamina propria and smooth muscle layers, forming a network; (ii) PDGFR $\alpha^{+}$cells were located between SMCs and were surrounded with smooth muscle bundles; (iii) PDGFR $\alpha^{+}$cells 


\section{Articles $\mid$ Hunziker et al.}
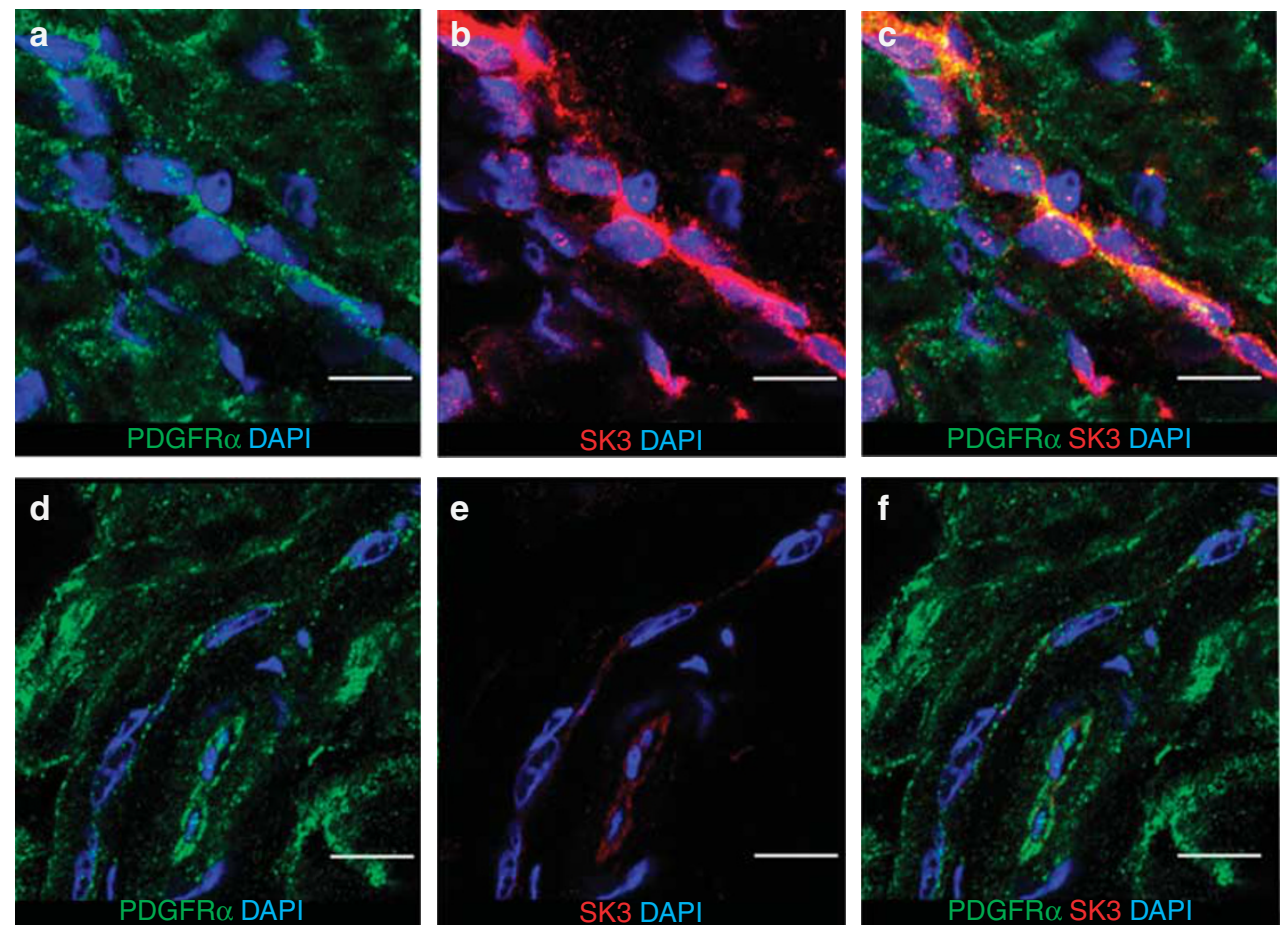

Figure 3. Expression of SK3. Double immunolabeling of PDGFRa (green) and SK3 (red) in the muscle layers of the human UPJ control (a-c) and UPJ obstruction (d-f). SK3 was markedly decreased in UPJ obstruction (e) compared with controls (b). Nuclei were stained with DAPI (blue) in all panels. $\mathrm{Bar}=10 \mu \mathrm{m}$ in all panels. DAPI, 4',6-diamidino-2-phenylindole; PDGFRa, platelet-derived growth factor receptor a; UPJ, ureteropelvic junction.
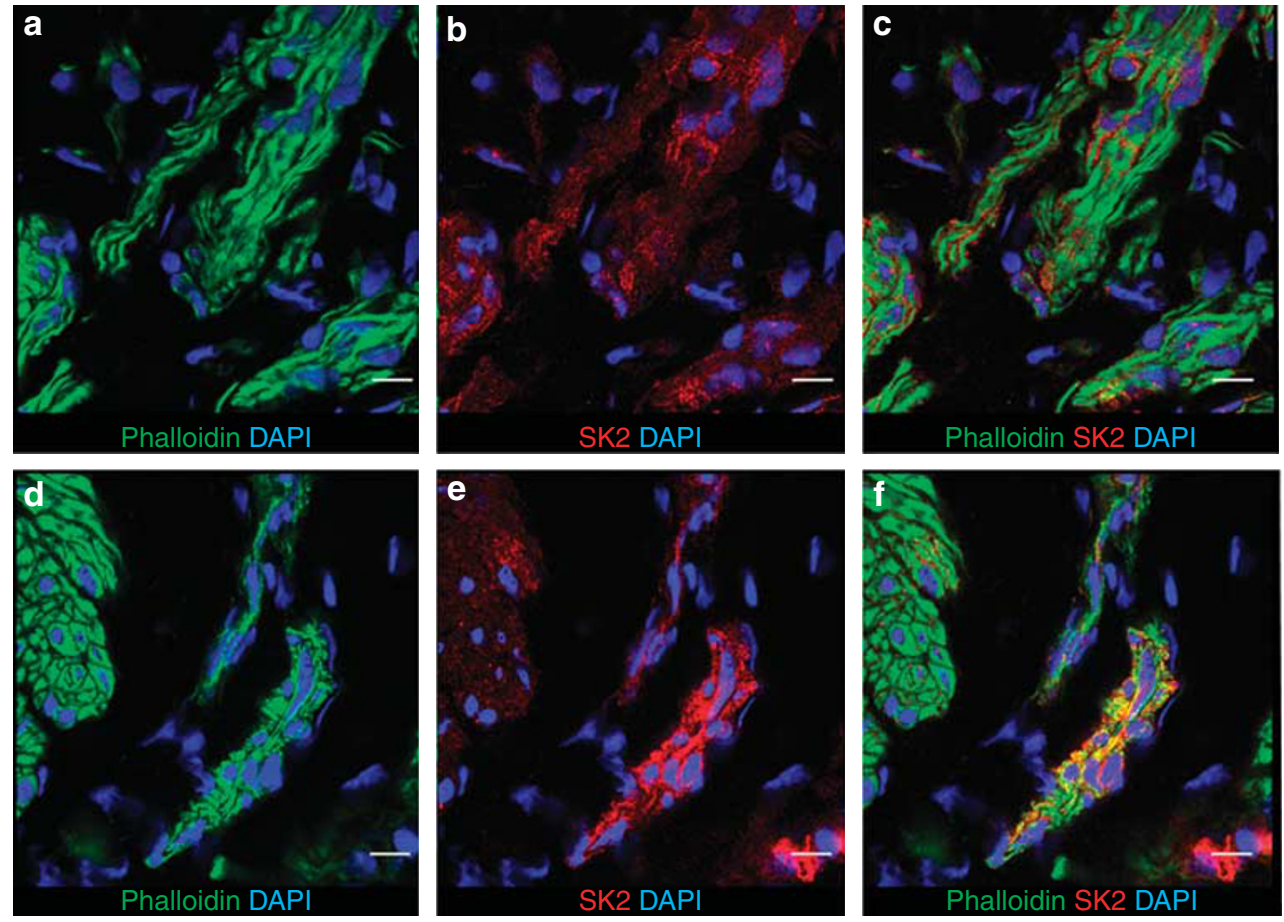

Figure 4. Expression of SK2. Double immunolabeling of phalloidin (green) and SK2 (red) in the muscle layers of the human UPJ control (a-c) and UPJ obstruction (d-f). SK2 was markedly increased in UPJ obstruction (e) compared with controls (b). Nuclei were stained with DAPI (blue) in all panels. Bar $=10 \mu \mathrm{m}$ in all panels. DAPI, 4',6-diamidino-2-phenylindole; UPJ, ureteropelvic junction. 


\section{PDGFRa ${ }^{+}$cells in the human UPJ Articles}

a1

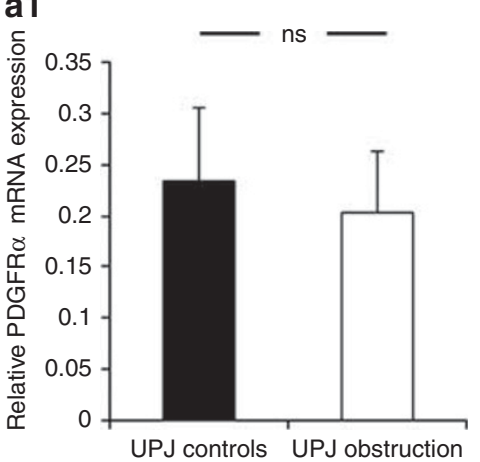

b1
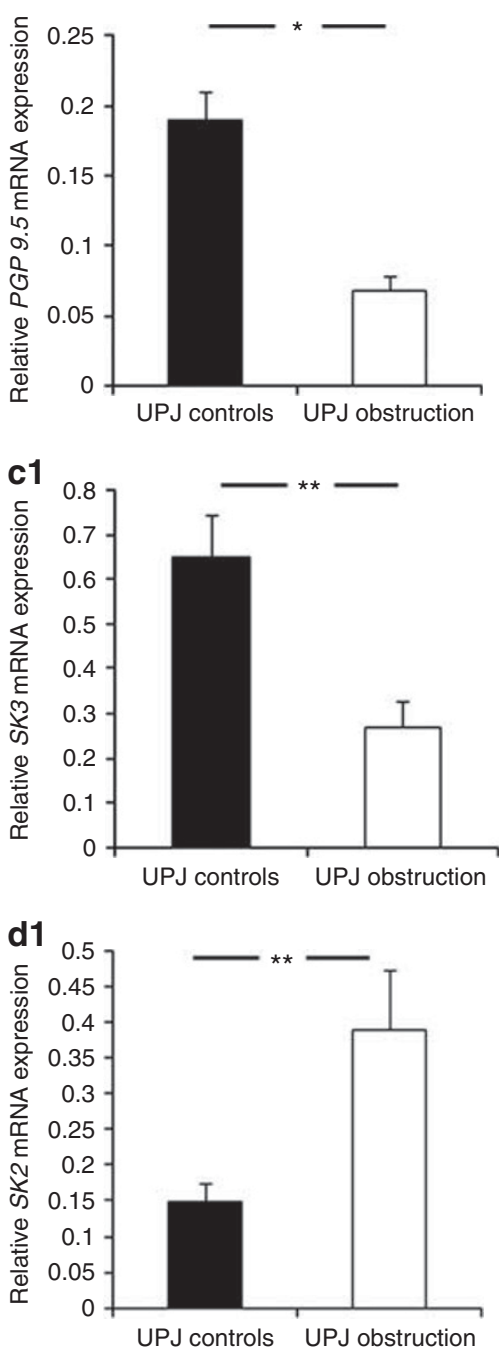

a2

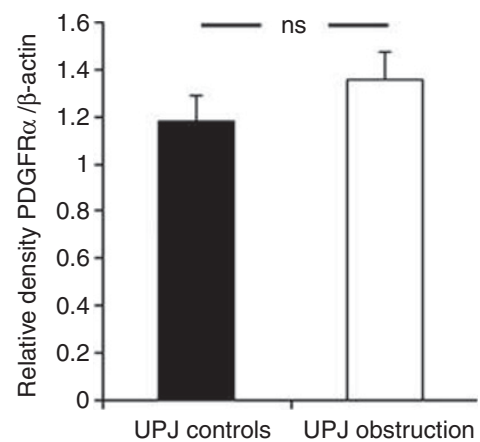

b2

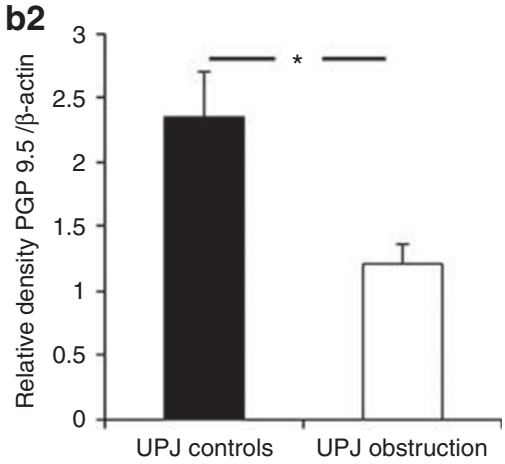

c2

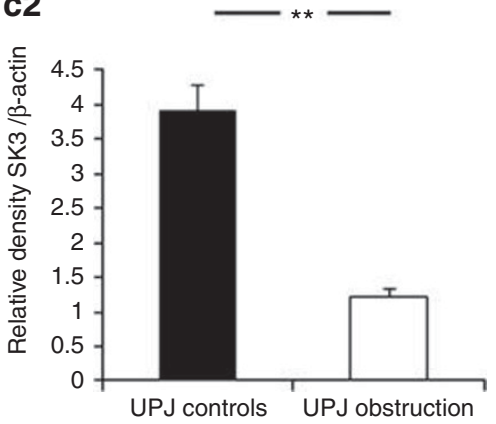

d2

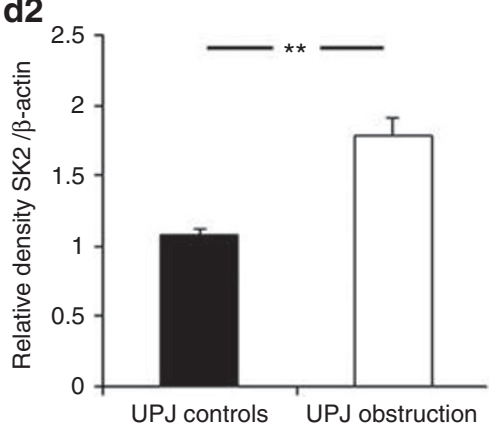

a3

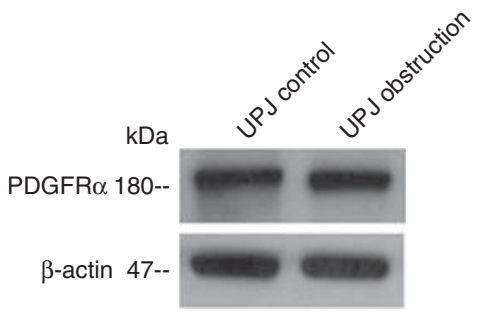

b3

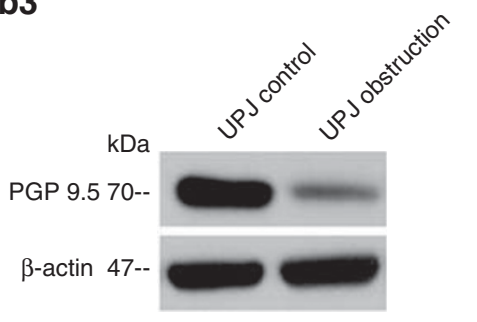

c3

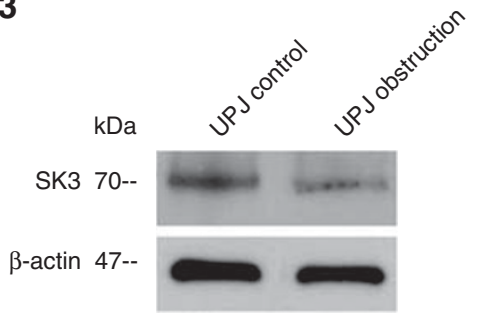

d3

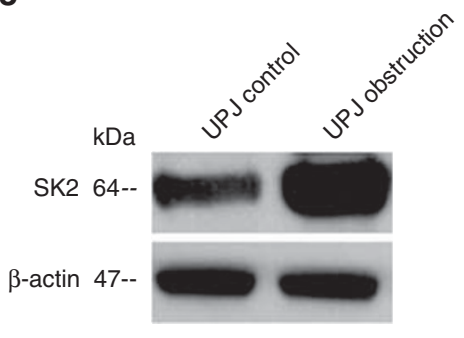

Figure 5. Relative mRNA expression levels and western blot. Relative mRNA expression levels, western blot, and densitometry of PDGFRa (a1, a2, a3), PGP9.5 (b1, b2, b3), SK3 (c1, c2, c3), and SK2 (d1, d2, d3). Relative mRNA expression levels of PDGFRa were similar between UPJ controls and UPJ obstruction $(\mathbf{a} 1 ; P=0.566)$. Densitometry analysis $(\mathbf{a} 2)$ of western blots $(\mathbf{a} 3)$ showed no difference in protein expression levels of PDGFRa in UPJ obstruction compared with controls. Relative mRNA expression levels of PGP9.5 were significantly decreased in UPJ obstruction compared with controls $(\mathbf{b} 1 ; P<0.001)$. Densitometry analysis $(\mathbf{b} 2)$ of western blots $(\mathbf{b} 3)$ showed a semiquantitative decrease in protein levels of PGP9.5 in UPJ obstruction compared with controls. Relative mRNA expression levels of SK3 were significantly decreased in UPJ obstruction compared with controls $(\mathbf{c} 1 ; P=0.016)$. Densitometry analysis (c2) of western blots (c3) showed a semiquantitative decrease in protein levels of SK3 in UPJ obstruction compared with those in controls. Relative mRNA expression levels of SK2 were significantly increased in UPJ obstruction compared with controls (d1; $P=0.017$ ). Densitometry analysis (d2) of western blots (d3) showed a semi-quantitative increase in protein levels of SK2 in UPJ obstruction compared with controls. Results are presented as mean \pm SEM $\left({ }^{*} P \leq 0.05,{ }^{*} P \leq 0.005\right)$. PDGFRa, platelet-derived growth factor receptor $a$; PGP9.5, pan-neuronal marker protein gene product 9.5; UPJ, ureteropelvic junction. 


\section{Articles $\mid$ Hunziker et $a$ al.}

were closely associated with, but were distinct from c-kitpositive IC-LCs; (iv) PDGFR $\alpha^{+}$cells were in close proximity to PGP9.5-positive nerve fibers; and (v) PDGFR $\alpha^{+}$cells expressed SK3 channels (17).

The neuronal network in UPJ has been described in previous studies $(4,11)$. We confirmed our previous finding of a decreased PGP9.5 mRNA transcript (3), and now also found decreased PGP9.5 protein expression levels in UPJ obstruction compared with controls. Defective innervation has previously been suggested to have an important role in the pathogenesis of UPJ obstruction $(3,18)$.

The main aim of our study was to investigate whether the PDGFR $\alpha$ antibody characterizes a novel population of interstitial cells in the upper urinary tract, and whether these interstitial cells are different from c-kit-positive IC-LCs. Our findings confirm that PDGFR $\alpha^{+}$cells represent a new class of interstitial cells in the upper urinary tract. Interstitial cells in the upper urinary tract can, therefore, be divided into at least two groups: (i) C-kit-positive interstitial cells (IC-LCs) and (ii) PDGFR $\alpha^{+}$interstitial cells. Our findings are similar to those reported in the gastrointestinal tract where ICCs and PDGFR $\alpha^{+}$cells represent two different cell types $(9,19)$.

The close proximity of PDGFR $\alpha^{+}$cells and ICCs to SMCs is well known in the gastrointestinal tract where all three cell types-(i) SMCs, (ii) ICCs, and (iii) PDGFR $\alpha^{+}$cells form the SMCs-ICCs-PDGFR $\alpha+$ cell (SIP) syncytium (9). In the gastrointestinal tract, ICCs can generate spontaneous electrical activity. Spontaneous electrical activity conducts to SMCs and drives electrical slow waves and phasic contractions. Contractile behavior is modulated by ICCs and PDGFR $^{+}$cells as these cells are able to conduct neural inputs to SMCs (9).

In the upper urinary tract, IC-LCs are thought to have a crucial role in facilitating pyeloureteric peristalsis (2). Studies have shown that IC-LCs are responsible for producing and coordinating ureteropelvic peristaltic movements at the intercalyceal area to promote the passage of urine from the renal pelvis through the ureter to the bladder (2). IC-LCs, which are located between neurons, atypical SMCs, and SMCs, are responsible for the amplification and conduction of slow wave electrical potentials for peristaltic movements (2). However, atypical SMCs have been argued to be more directly involved in generating the membrane potential fluctuations underlying pacemaking $(2,20)$.

Potassium channels have been well explored in the upper urinary tract in the guinea pig (21). Several types of $\mathrm{K}^{+}$ channels are thought to participate in the regulation of smooth muscle excitability in the urinary tract. Two families of $\mathrm{Ca}^{2+}$-activated $\mathrm{K}^{+}$conductances have been characterized in bladder SMCs: large-conductance (BK) and SK channels (13). SK channels are a family of three subtypes, SK1-SK3. SK channels are insensitive to the changes in the membrane potential and are exclusively activated by rises of the intracellular $\mathrm{Ca}^{2+}$ (ref. 22). The activation of SK channels results in $\mathrm{K}^{+}$efflux causing hyperpolarization of muscles and muscle relaxation (14). It has furthermore been shown that SK channels have an important role in shaping and regulating the frequency of urinary bladder smooth muscle action potentials, thereby modulating contractility (15). In the guinea pig upper urinary tract, SK channels are involved in the repolarization of action potentials (21). In addition, it has been shown that a positive modulation of SK channels can lower blood pressure in animal models of hypertension. Thus, SK channels have been demonstrated as potent therapeutic targets for the treatment of hypertension (23). However, to our knowledge, no study has thoroughly investigated the effects of potential SK channel therapeutics in the upper urinary tract.

Our study was able to demonstrate the expression of the SK3 channel protein in PDGFR $\alpha^{+}$cells of the UPJ. Our findings are in line with previous studies of the human and murine gastrointestinal tract $(14,24)$ and murine bladder (13), showing SK3 channel expression in PDGFR $\alpha^{+}$cells.

Recent detailed morphological and functional studies of murine detrusor muscles not only confirmed the expression of SK3 channel in PDGFR $\alpha^{+}$cells, but also measured singlechannel currents consistent with the conductance and $\mathrm{Ca}^{2+}$ sensitivity of SK3 channels in PDGFR $\alpha^{+}$cells (13). Further studies by the same group investigated responses of PDGFR $\alpha^{+}$ cells to ATP. Their data suggest that purines can activate SK currents in PDGFR $\alpha^{+}$cells (25). Collectively, inhibitory neurotransmission in the bladder is mediated via purinergic mechanisms through PDGFR $\alpha^{+}$interstitial cells (25).

Although our study showed that the overall expression of PDGFR $\alpha^{+}$cells was not altered in UPJ obstruction compared with controls, SK3 was decreased in PDGFR $\alpha^{+}$cells in UPJ obstruction. Furthermore, SK3 mRNA transcripts and protein expression levels were decreased in the obstructed segment in UPJ obstruction. Blockade of SK 3 channels has been shown to depolarize the membrane potential of PDGFR cells and to increase the tension of the neighboring interconnected smooth muscle cells (26). Similarly, a decrease in SK3 channels in UPJ obstruction could lead to increased contractility of smooth muscle cells resulting in impaired peristalsis.

SK2 channels, on the other hand, were not expressed in PDGFR $\alpha^{+}$cells, but were found in SMCs. Similarly, Thorneloe et al. (15) demonstrated the expression of SK2 in urinary bladder smooth muscle. We also found expression of SK2 in SMCs as well as increased SK2 mRNA transcripts and overall SK2 protein expression levels in UPJ obstruction compared with controls. Thorneloe et al. (15) showed that SK2 was the essential functional subtype of SK channels in mouse urinary bladder SMCs. Selective blockade of SK2 channels increased contractility indicating an inhibitory role of SK2 for smooth muscle functioning (15). Collectively, the increased SK2 expression in UPJ obstruction suggests that SK2 channels could have a role in the disturbance of coordinated contraction of SMCs, which may cause impaired peristalsis in intrinsic UPJ obstruction. It is, however, not clear whether developmental abnormalities of SK channels have a primary or secondary role in congenital UPJ obstruction. 
A limitation of the study is the use of the UPJ of patients who have Wilm's tumor as a control group, which could potentially affect the results. Because it is difficult to find normal UPJ tissue in an adequate number of children as a control group, we believe that Wilm's tumor is the main, if not the only option, to obtain the unaffected UPJ for the analysis in the control group.

In conclusion, our study describes, for the first time, the presence and distribution of PDGFR $\alpha^{+}$cells that form a discrete network in the human UPJ. PDGFR $\alpha^{+}$cells, IC-LCs, and SMCs might form a syncytium in the upper urinary tract that may have an important role in motor neurotransmission and peristaltic contraction mechanisms. Further investigation of this novel class of interstitial cells in the upper urinary tract will provide new insights into the pathophysiology of UPJ obstruction.

\section{ACKNOWLEDGMENTS}

This work was funded by the National Children's Research Centre/ Children's Medical Research Foundation, Our Lady's Children's Hospital Crumlin, Dublin, Ireland. We thank Luiz Alvarez and Nicolae Corcionivoschi for technical assistance. We thank Feargal Quinn, Sri Paran, and Brice Antao, Department of Pediatric Surgery in Our Lady's Children's Hospital Crumlin for providing samples. We wish to acknowledge Michael, McDermott, and Maureen O'Sullivan, Department of Histopathology in Our Lady's Children's Hospital Crumlin for their assistance in specimen collection.

\section{STATEMENT OF FINANCIAL SUPPORT}

This study was funded by the Children's Medical and Research Foundation, Our Lady's Children's Hospital Crumlin, Dublin, Ireland.

Disclosure: The authors declare no conflict of interest.

\section{REFERENCES}

1. Gosling JA, Dixon JS. Functional obstruction of the ureter and renal pelvis. A histological and electron microscopic study. Br J Urol 1978;50: $145-52$.

2. Di Benedetto A, Arena S, Nicotina PA, Mucciardi G, Gali A, Magno C. Pacemakers in the upper urinary tract. Neurourol Urodyn 2013;32:349-53.

3. Wang Y, Puri P, Hassan J, Miyakita H, Reen DJ. Abnormal innervation and altered nerve growth factor messenger ribonucleic acid expression in ureteropelvic junction obstruction. J Urol 1995;154:679-83.

4. Murakumo M, Nonomura K, Yamashita T, Ushiki T, Abe K, Koyanagi T. Structural changes of collagen components and diminution of nerves in congenital ureteropelvic junction obstruction. J Urol 1997;157:1963-8.

5. Solari V, Piotrowska AP, Puri P. Altered expression of interstitial cells of Cajal in congenital ureteropelvic junction obstruction. J Urol 2003;170 (6 Pt 1): 2420-.

6. Lang RJ, Davidson ME, Exintaris B. Pyeloureteral motility and ureteral peristalsis: essential role of sensory nerves and endogenous prostaglandins. Exp Physiol 2002;87:129-46.

7. Popescu LM, Faussone-Pellegrini MS. TELOCYTES - a case of serendipity: the winding way from Interstitial Cells of Cajal (ICC), via Interstitial Cajal-Like Cells (ICLC) to TELOCYTES. J Cell Mol Med 2010;14:729-40.

8. McCloskey KD. Interstitial cells in the urinary bladder-localization and function. Neurourol Urodyn 2010;29:82-7.
9. Sanders KM, Ward SM, Koh SD. Interstitial cells: regulators of smooth muscle function. Physiol Rev 2014;94:859-907.

10. Iino S, Horiguchi K, Horiguchi S, Nojyo Y. c-Kit-negative fibroblast-like cells express platelet-derived growth factor receptor alpha in the murine gastrointestinal musculature. Histochem Cell Biol 2009;131: 691-702.

11. Monaghan KP, Johnston L, McCloskey KD. Identification of PDGFRalpha positive populations of interstitial cells in human and guinea pig bladders. J Urol 2012;188:639-47.

12. Koh BH, Roy R, Hollywood MA, et al. Platelet-derived growth factor receptor-alpha cells in mouse urinary bladder: a new class of interstitial cells. J Cell Mol Med 2012;16:691-700.

13. Lee H, Koh BH, Peri LE, Sanders KM, Koh SD. Functional expression of SK channels in murine detrusor PDGFR+ cells. J Physiol 2013;591: 503-13.

14. Kurahashi M, Mutafova-Yambolieva V, Koh SD, Sanders KM. Plateletderived growth factor receptor-alpha-positive cells and not smooth muscle cells mediate purinergic hyperpolarization in murine colonic muscles. Am J Physiol Cell Physiol 2014;307:C561-70.

15. Thorneloe KS, Knorn AM, Doetsch PE, et al. Small-conductance, $\mathrm{Ca}(2+)$ -activated $\mathrm{K}+$ channel 2 is the key functional component of SK channels in mouse urinary bladder. Am J Physiol Regul Integr Comp Physiol 2008;294:R1737-43.

16. Gevaert T, De Vos R, Van Der Aa F, et al. Identification of telocytes in the upper lamina propria of the human urinary tract. J Cell Mol Med 2012;16: 2085-93.

17. Nemeth L, O'Briain DS, Puri P. Demonstration of neuronal networks in the human upper urinary tract using confocal laser scanning microscopy. J Urol 2001;166:255-8.

18. Kajbafzadeh AM, Payabvash S, Salmasi AH, Monajemzadeh M, Tavangar SM. Smooth muscle cell apoptosis and defective neural development in congenital ureteropelvic junction obstruction. J Urol 2006;176:718-23.

19. Peri LE, Sanders KM, Mutafova-Yambolieva VN. Differential expression of genes related to purinergic signaling in smooth muscle cells, PDGFRalpha-positive cells, and interstitial cells of Cajal in the murine colon. Neurogastroenterol Motil 2013;25:e609-20.

20. Klemm MF, Exintaris B, Lang RJ. Identification of the cells underlying pacemaker activity in the guinea-pig upper urinary tract. J Physiol 1999;519 (Pt 3): 867-4.

21. Lang RJ, Zhang Y. The effects of $\mathrm{K}+$ channel blockers on the spontaneous electrical and contractile activity in the proximal renal pelvis of the guinea pig. J Urol 1996;155:332-6.

22. Parajuli SP, Hristov KL, Soder RP, Kellett WF, Petkov GV. NS309 decreases rat detrusor smooth muscle membrane potential and phasic contractions by activating SK3 channels. Br J Pharmacol 2013;168: 1611-25.

23. Cui M, Qin G, Yu K, Bowers M, Zhang M. Targeting the small- and intermediate-conductance Ca-activated potassium channels: the drugbinding pocket at the channel/calmodulin interface. Neurosignals 2014;22:65-78.

24. Kurahashi M, Nakano Y, Hennig GW, Ward SM, Sanders KM. Plateletderived growth factor receptor alpha-positive cells in the tunica muscularis of human colon. J Cell Mol Med 2012;16:1397-404.

25. Lee H, Koh BH, Peri LE, Sanders KM, Koh SD. Purinergic inhibitory regulation of murine detrusor muscles mediated by PDGFRalpha+ interstitial cells. J Physiol 2014;592:1283-93.

26. Herrera GM, Pozo MJ, Zvara P, et al. Urinary bladder instability induced by selective suppression of the murine small conductance calciumactivated potassium (SK3) channel. J Physiol 2003;551:893-903. 